\title{
PUNÇÃO EM LAJES COGUMELO DE CONCRETO ARMADO - RETANGULARIDADE DOS PILARES
}

\author{
PUNCHING SHEAR IN REINFORCED CONCRETE FLAT SLABS - \\ COLUMN RECTANGULARITY
}

\author{
Valéria Conceição Mouro Costa ${ }^{1}$, Diorgenes Carvalho de Oliveira ${ }^{2}$, Ronaldo Barros Gomes ${ }^{3}$, \\ Gilson Natal Guimarães ${ }^{4}$
}

Recebido em 29 de agosto de 2012; recebido para revisão em 05 de setembro de 2012; aceito em 18 de setembro de 2012; disponível on-line em 16 de outubro de 2012.

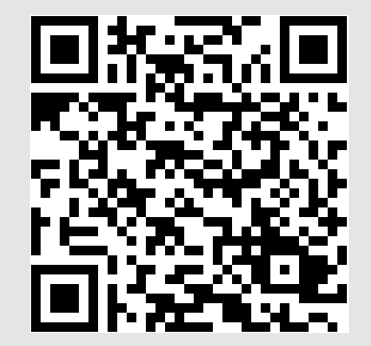

PALAVRAS CHAVES:

Laje cogumelo;

Concreto armado;

Punção;

Pilar retangular.

\section{KEYWORDS:}

Flat Slab;

Reinforced Concrete;

Punching;

Rectangular column.

\begin{abstract}
RESUMO: Foram ensaiadas e analisadas 8 (oito) lajes cogumelo de concreto armado quadradas com $1800 \mathrm{~mm}$ de lado e $130 \mathrm{~mm}$ de espessura, submetidas a carregamento central, com o objetivo de investigar a influência do índice de retangularidade do pilar, sendo que a relação entre as dimensões da seção transversal do pilar $\left(c_{\text {máx }} / c_{\text {min }}\right)$ variou entre 1 e 4, com perímetro mantido constante e igual a $1000 \mathrm{~mm}$. São apresentados e analisados os resultados das cargas de ruptura, modos de ruptura, deslocamentos verticais, fissuração e deformações do aço e concreto. São apresentadas comparações dos resultados experimentais com códigos, normas de projeto e resultados da literatura. Os resultados indicam que à medida que aumenta o índice de retangularidade, a resistência à punção diminui para lajes que possuem pilares com o perímetro constante, sugerindo que o combate à punção pode ser mais efetivo em regiões próximas das extremidades dos pilares, onde há maior concentração de forças cortantes. É apresentada uma proposta de inclusão do parâmetro $c_{\operatorname{máx}} / c_{\min }$ nas expressões de cálculo da resistência à punção das normas ACl (2002), CEB-FIP (1991), EC2 (2001) e NBR-6118 (2003).
\end{abstract}

ABSTRACT: Eight reinforced concrete flat slabs were tested. Slabs were $1800 \mathrm{~mm}$ square and $130 \mathrm{~mm}$ thick and were submitted to concentric loading. The objective of this study was to investigate the influence of column rectangularity coefficient. The relationship between the column cross section dimensions $\left(c_{\max } / c_{\min }\right)$ varyied between 1 and 4 with a constant perimeter equal to $1000 \mathrm{~mm}$. Results presented and analyzed include ultimate loads and failure types, modes of rupture, vertical displacements, cracking and deformations of steel and concrete. Comparisons with codes and with results obtained by other researchers are also presented. The results indicate that as the rectangularity coefficient increases, punching shear resistance decreases for constant perimeter column flat slabs. This suggests that punching shear has to be considered especially in regions close to column corners, where there is a larger concentration of shear forces. A proposal for an inclusion of the rectangularity parameter $\left(c_{\text {máx }} / c_{\text {min }}\right)$ in expressions for calculation punching shear resistance of ACl (2002), CEB-FIP (1991), EC2 (2001) and NBR-6118 (2003) codes is presented.

\footnotetext{
* Contato com os autores:

${ }^{1}$ e-mail : valeriamouro@gmail.com (V. C. M. Costa)

Enga. Civil mestranda do curso de Pós-Graduação em Engenharia Civil da Escola de Engenharia Civil - CMEC / UFG

${ }^{2}$ e-mail : diordrum@gmail.com (D. C. de Oliveira)

Enga. Civil mestrando do curso de Pós-Graduação em Engenharia Civil da Escola de Engenharia Civil - CMEC / UFG

3e-mail : rbggomes@gmail.com (R. B. Gomes)

Prof. Dr. da Escola de Engenharia Civil - Universidade Federal de Goiás - UFG.

${ }^{4}$ e-mail : gilson@eec.ufg.br (G. N. Guimarães)

Prof. Dr. da Escola de Engenharia Civil - Universidade Federal de Goiás - UFG.
} 


\section{INTRODUÇÃO}

Segundo a NBR 6118 (2003), lajes-cogumelo são lajes apoiadas diretamente em pilares com capitéis, enquanto lajes lisas são apoiadas nos pilares sem capitéis. Estas placas estão sujeitas à ruptura por punção na ligação laje-pilar, que ocorre frágil e repentinamente. Punção é o estado limite último determinado por cisalhamento no entorno de forças concentradas. É decorrente da grande concentração de tensões na região da ligação laje-pilar, a qual está submetida tanto ao esforço cortante como ao momento fletor negativo máximo.

A literatura apresenta diversos trabalhos que estudam o comportamento da ligação de lajes cogumelo com pilares de seção transversal quadrada, sendo a pesquisa com pilares retangulares em menor número, como os trabalhos de Hawkins et al. (1971), Regan e Rezai-Jorabi (1988), Teng et al. (1999), Al-Yousif e Regan (2003), Silva (2003), Oliveira (2003) e Borges (2004), apesar da sua utilização prática ser mais frequente. 0 presente trabalho aborda o estudo da influência da retangularidade da seção transversal dos pilares, com a variação das dimensões dos lados do pilar, e perímetro constante, na resistência à punção das lajes cogumelo de concreto armado.

\subsection{OBJETIVO E JUSTIFICATIVA}

Os principais objetivos deste estudo são:

- Investigar o comportamento último e em serviço à punção de lajes cogumelo de concreto armado submetidas ao carregamento central, com índices de retangularidade variando entre 1 e 4, e o perímetro dos pilares igual a 1000 $\mathrm{mm}$;

- Analisar as normas e códigos de projeto visando melhorar a expressão de cálculo da resistência à punção de lajes cogumelos considerando pilares retangulares;

- Propor a inclusão do parâmetro $\mu$, que leva em consideração a influência da relação entre a maior e a menor dimensão do pilar $\left(c_{\max } / c_{\min }\right)$, nas expressões de cálculo das normas ACl-318 (2002), CEB-FIP MC90 (1991), EUROCODE 2 (2001), NBR-6118 (2003).

As pesquisas realizadas sobre as ligações de lajes com pilares retangulares ainda não resultaram em um modelo de dimensionamento à punção capaz de satisfazer a todos os questionamentos em relação a essas ligações. Encontram-se poucos ensaios envolvendo lajes cogumelo com pilares retangulares na literatura.

\section{REVISÃO BIBLIOGRÁFICA}

Segundo Cordovil (1995), a norma americana ACl desde 1963 abordava o problema de punção em placas de concreto armado. $\mathrm{Na}$ atual versão, a $\mathrm{ACl}$ 318/02 considera o índice de retangularidade dos pilares. As normas CEB-FIP MC90 (1991), EUROCODE 2 (2001) e a NBR 6118 (ABNT, 2003) não levam em consideração a geometria dos pilares na resistência à punção de lajes cogumelo.

A seção crítica, ou de controle, de lajes sujeitas à punção, está localizada a uma distância medida a partir da face do pilar ou da área carregada. Para uma laje cogumelo com altura efetiva " $d$ " apoiada em pilar de seção retangular com dimensões $c_{\min }$ (menor dimensão) e $c_{\text {máx }}$ (maior dimensão), o perímetro de controle é obtido conforme mostra a Figura 1, para as normas do $\mathrm{ACl} 318$ (2002), sendo igual para as normas CEB-FIP MC90 (1991), EUROCODE 2 (2001) e NBR 6118 (ABNT, 2003).

1318

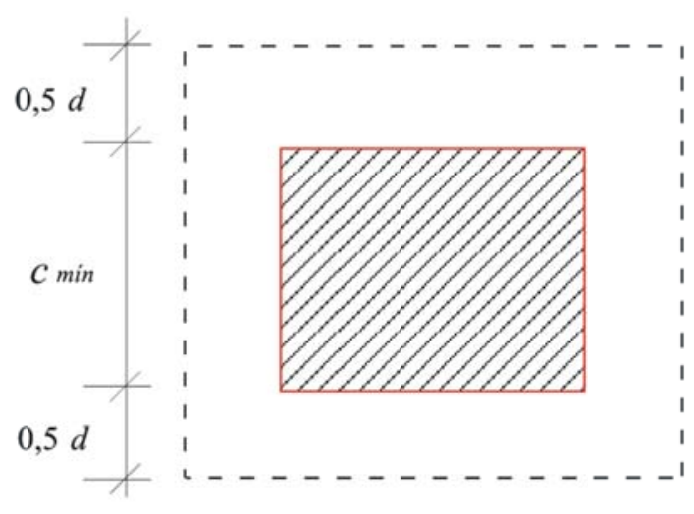

MC90 (1991), NT, 2003):

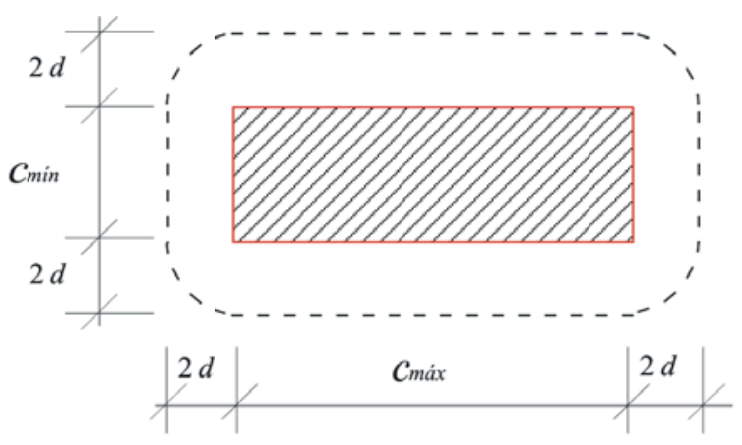

Figura 1: Perímetro de controle de acordo com as normas de projeto. Fontes: ACI 318 (2002), CEB-FIP MC90 (1991), EUROCODE 2 (2001), NBR 6118 (ABNT, 2003).

A verificação da punção em lajes cogumelo, segundo ACl 318 (2002) é realizada através da comparação entre a força nominal atuante $V$ e a força 
nominal resistente $V_{n}$. Para uma laje sem armadura de cisalhamento, a força nominal resistente é oferecida apenas pelo concreto $V_{c}$, dada pela Equação 1 .

$$
V \leq V_{c} \quad(\mathrm{kN}) \quad \text { Eq. }[01]
$$

Onde:

$V_{c}=$ é o menor valor obtido através das Equações 2, 3 e 4.

$$
\mathrm{v}_{\mathrm{c}}<\left\{\begin{array}{ccc}
\left(1+\frac{2}{\beta_{c}}\right) \frac{1}{6} \sqrt{f_{c}^{\prime} b_{0} d} & (\mathrm{kN}) & \text { Eq. [02] } \\
\left(\frac{\alpha_{s} d}{b_{0}}+2\right) \frac{1}{12} \sqrt{f_{c}^{\prime}} b_{0} d & (\mathrm{kN}) & \text { Eq. [03] } \\
\frac{1}{3} \sqrt{f_{c}^{\prime}} b_{0} d & \text { (kN) } & \text { Eq. [04] }
\end{array}\right.
$$

Onde:

$\beta_{c}=$ relação entre o lado maior e o lado menor do pilar;

$\boldsymbol{f}_{c}^{\prime}=$ resistência à compressão do concreto em corpos de prova cilíndricos, em MPa;

$\boldsymbol{b}_{\boldsymbol{0}}=$ perímetro de controle localizado a $0,5 \mathrm{~d}$ do pilar, em $\mathrm{mm}$;

$\boldsymbol{d}=$ altura efetiva da laje, em $\mathrm{mm}$;

$\alpha_{s}=40$ (pilares internos), 30 (pilares de borda) e 20 (pilares de canto).

O valor obtido da Equação 2 se torna não conservativo quando se tem relações entre os lados do pilar com valores maiores que 2, tratando-se, neste caso, de pilares retangulares. Para estes pilares, a tensão real de cisalhamento na seção de controle, atinge o valor máximo dado pela Equação 2 apenas na região dos cantos do pilar, diminuindo este valor em direção à região central do lado do pilar.

Ensaios realizados constataram ainda que, na medida em que o valor da razão $b_{0} / d$ aumenta, nos casos de pilares de grandes dimensões, o valor de $v_{c}(=$ $\left.V_{d} / b_{0} d\right)$ diminuía. Baseados nestes aspectos é que foram desenvolvidas as Equações 2 e 3, de forma a se levar em consideração os fatores mencionados no cálculo de $v_{c}$. As Equações 3 e 4 mostram que o método de cálculo do $\mathrm{ACl} 318$ (2002) trata o caso de pilar retangular através da redução das tensões nominais cisalhantes, e desconsidera a influência da armadura de flexão e o efeito de tamanho na resistência ao puncionamento.
O CEB-FIP MC90 (1991) não apresenta consideração quando os pilares ou áreas carregadas apresentam, para relações entre lado maior e lado menor, valores superiores a 2. Esta norma considera para as ligações laje-pilar que não possuem armadura de punção, que sejam feitas duas verificações:

- Verificação da compressão do concreto, através da comparação das tensões atuantes na seção de controle definida pelo pilar e a tensão resistente;

- Comparação entre a tensão nominal atuante no perímetro de controle localizado a $2 \mathrm{~d}$ do contorno do pilar, com uma tensão nominal resistente.

A altura efetiva $d$ é calculada pela média aritmética das alturas efetivas nas direções ortogonais $x$ e $y$ conforme mostra a equação 5 .

$$
d=\frac{d_{x}+d_{y}}{2} \quad(\mathrm{~mm})
$$

A taxa geométrica de armadura principal de flexão $\rho$ é obtida pela média geométrica das taxas de armadura $\rho_{x}$ e $\rho_{y}$ nas direções ortogonais $x$ e $y$, respectivamente, em uma região com largura igual ao lado do pilar mais $3 d$ para cada lado, sendo dada pela Equação 6.

$$
\rho=\sqrt{\rho_{x} \rho_{y}} \quad \text { (\%) } \quad \text { Eq. [06] }
$$

Na verificação da seção de controle localizada a $2 d$ do pilar, a tensão de cisalhamento atuante na seção de controle $v$ é comparada com a tensão de cisalhamento resistente $v_{n}$ como mostra a Equação 7 .

$$
v \leq v_{n}
$$

A tensão de cisalhamento resistente de lajes sem armadura de cisalhamento, dada apenas pela contribuição do concreto $v_{c}$, é obtida pela Equação 8.

$$
v_{c}=v_{s d} \leq 0,12\left(1+\sqrt{\frac{200}{d}}\right)\left(100 \rho f_{c k}\right)^{1 / 3} u d \text { (MPa) Eq. [08] }
$$

\section{Onde:}

$\mathbf{u}=$ é o perímetro de controle situado a $2 \mathrm{~d}$ do pilar, em $\mathrm{mm}$;

$\rho=$ é a taxa geométrica da armadura principal de flexão, em \%.

\section{O EUROCODE 2 (2001) apresenta} recomendações muito semelhantes ao do CEB-FIP MC90 (1991), com relação às verificações da seção de 
controle: verificação da tensão máxima de cisalhamento no perímetro do pilar $\left(v \leq v_{\text {máx }}\right)$, da tensão de cisalhamento atuante na seção de controle a $2 d$ do pilar $\left(v \leq v_{n}\right)$, e da tensão de cisalhamento atuante na seção de controle a $1,5 d$ da última camada de armadura de cisalhamento, se houver. A tensão de cisalhamento atuante $v$ é dada pela Equação 9, onde $u_{1}$ é o perímetro de controle a $2 d$ do pilar.

$$
v=\frac{v}{u_{1} d}
$$

(MPa) Eq. [09]

Para lajes sem armadura de cisalhamento, são feitas duas verificações: na face do pilar, através da Equação 10 , e na seção de controle a $2 d$ do pilar, através da equação 11.

$$
\begin{gathered}
v=\frac{v}{u_{0} d} \leq v_{\text {máx }}=0,5 v f_{c k} \quad \text { (MPa) } \\
v=\frac{v_{s d}}{u_{1} d}=\frac{0,18}{\gamma_{c}}\left(1+\sqrt{\frac{200}{d}}\right)\left(100 \rho_{1} f_{c k}\right)^{1 / 3} \quad \text { (10] }
\end{gathered}
$$

\section{Onde:}

$\boldsymbol{u}_{0}=$ é o perímetro do pilar ou da área carregada, em $\mathrm{mm}$;

$\boldsymbol{v}_{\text {máx }}=$ é a tensão máxima de cisalhamento atuante na face do pilar, em MPa;

$\boldsymbol{v}=$ é a resistência à compressão para um concreto fissurado, dada por $0,6\left(1-\frac{f_{c k}}{250}\right)$, em MPa;

$\boldsymbol{\rho}_{1}=\sqrt{\rho_{1 x} \rho_{1 y}} \leq 0,02$, é a taxa de armadura de flexão.

A NBR 6118 (ABNT, 2003) apresenta semelhanças em alguns conceitos com o CEB-FIP (1991), como por exemplo, na forma e definição dos perímetros de controle, e no cálculo das forças resistentes, variando apenas alguns coeficientes.

Em lajes sem armadura de punção, deve ser verificada a compressão diagonal do concreto na zona adjacente ao pilar, e a punção na superfície de controle distante $2 d$ da face do pilar. A verificação da compressão do concreto na seção do pilar é feita através da comparação entre a tensão atuante no perímetro do pilar $\tau_{s}$ e a tensão resistente $\tau_{r 2}$, conforme mostram as equações 12,13 e 14 .

$$
\begin{gathered}
\tau_{s} \leq \tau_{r 2} \\
\tau_{s}=\frac{V}{u_{0} d} \quad \text { (MPa) }
\end{gathered}
$$

\section{PROGRAMA EXPERIMENTAL}

O programa experimental visa representar uma situação semelhante à de edifícios residenciais com presença de lajes cogumelo apoiadas em pilares quadrados ou retangulares. Verifica-se neste trabalho a situação de punção, comum em pilares de centro de edifícios com lajes cogumelo com carregamento simétrico e sem excentricidade.

A Figura 2 mostra que o modelo adotado procurou representar uma região entre seções de momentos fletores nulos delimitada pela circunferência de raio $r \cong 1 / 5 L$, onde $L$ é o comprimento do vão de um painel.

Foram ensaiadas até a ruptura 8 lajes quadradas com $1800 \mathrm{~mm}$ de lado e $130 \mathrm{~mm}$ de espessura. A principal variável das lajes ensaiadas foi a dimensão do lado do pilar, com perímetro constante e igual a $1000 \mathrm{~mm}$. A relação entre o maior e o menor lado do pilar variou de 1 a 4 . A Figura 3 e a Tabela 1 apresentam as características geométricas das lajes.

O sistema de ensaio das lajes consistiu de um 


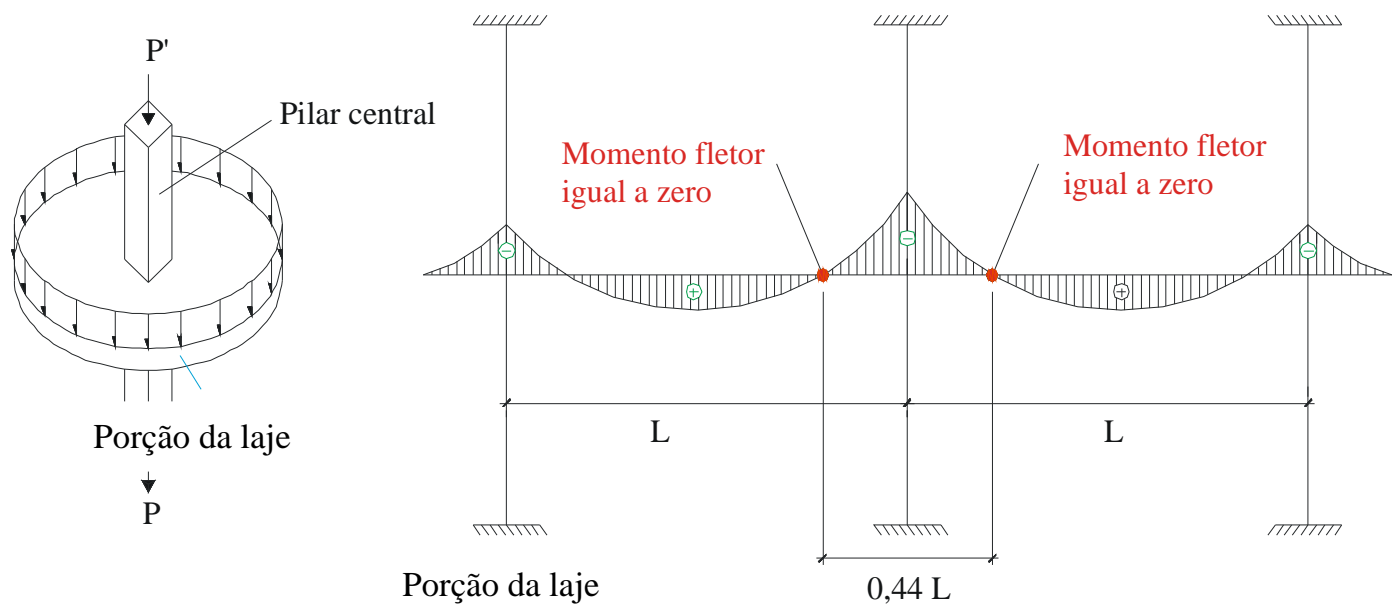

Figura 2: Modelo hipotético caracterizando a situação estudada.

conjunto de oito vigas metálicas, vigas 1 e 2, apoiadas em oito placas equidistantes formando uma circunferência de $825 \mathrm{~mm}$ de raio, que simulam os 8 pontos de carga, atirantadas em uma laje de reação. Estas placas tinham dimensões de $120 \times 200 \mathrm{~mm}$ e $25 \mathrm{~mm}$ de espessura. Uma representação detalhada do esquema de ensaio é mostrada nas Figuras 4 e 5.

A armadura de flexão foi igual para todas as lajes, adotando-se duas malhas, uma inferior e outra superior. A armadura de flexão negativa (malha superior) foi composta por 19 barras de $12,5 \mathrm{~mm}$ de diâmetro, espaçadas a cada $94 \mathrm{~mm}$, em cada direção. A ancoragem desta armadura foi feita por 19 ganchos de $6,3 \mathrm{~mm}$ de diâmetro, em forma de "U", em cada lado da laje. A armadura de flexão positiva (malha inferior) foi composta por 11 barras de $6,3 \mathrm{~mm}$ de diâmetro, espaçadas a $163 \mathrm{~mm}$, em cada direção.

Foram utilizados espaçadores de $15 \mathrm{~mm}$ de espessuras fixados à armadura de flexão positiva em todas as lajes, para definição do cobrimento de concreto. As placas que simularam os pilares foram dispostas com a maior dimensão paralela à direção y. O concreto utilizado para todas as lajes foi dosado para atingir uma resistência característica à compressão em torno de 25 $\mathrm{MPa}$, aos 28 dias.

Para determinar os deslocamentos verticais em alguns pontos da laje, utilizaram-se relógios comparadores digitais, com precisão de $0,01 \mathrm{~mm}$. As leituras dos deslocamentos foram efetuadas em duas direções perpendiculares, com a origem no centro da laje (sistema de coordenadas $x-y$ ). Foram posicionados relógios na linha dos pontos de inflexão de momento fletor, permitindo a obtenção dos deslocamentos da laje em relação à laje de reação.
Para medir a deformação das armaduras principais de flexão e no concreto, utilizou-se extensômetros elétricos de resistência (EER).

Todos os ensaios foram feitos com incrementos de carga de $25 \mathrm{kN}$ até atingir a ruptura. Em todas as cargas aplicadas foram feitas leituras de relógios, medição de trena no centro da laje, de extensômetros elétricos de resistência e de concreto. Observou-se a propagação das fissuras e fez a marcação das mesmas. Os ensaios das lajes tiveram duração de aproximadamente quatro horas.

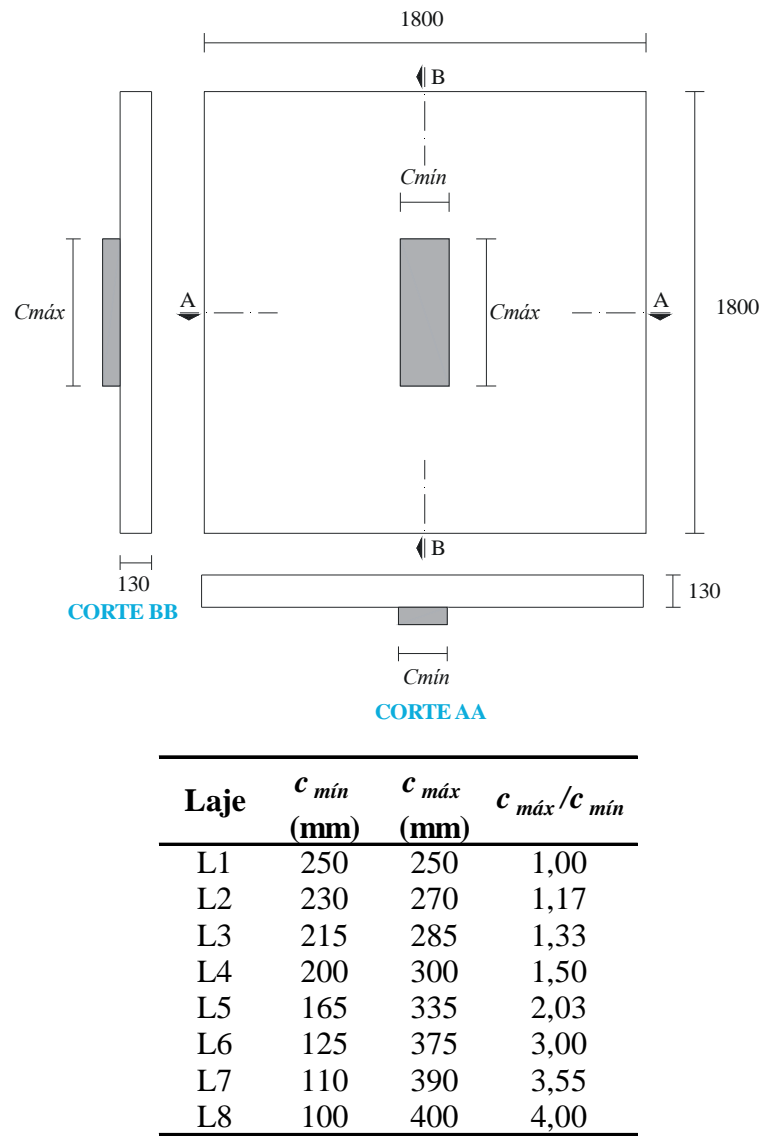

Figura 3: Características geométricas das lajes - $(\mathrm{mm})$. 
Tabela 1: Características geométricas das lajes ensaiadas

\section{Dimensões do pilar}

\begin{tabular}{|c|c|c|c|c|c|c|}
\hline \multirow[t]{2}{*}{ Laje } & \multicolumn{2}{|c|}{$\begin{array}{l}\text { Dimensões do pilar } \\
\text { (mm) }\end{array}$} & \multirow[t]{2}{*}{$c_{\text {máx }} / c_{\text {mín }}$} & \multirow{2}{*}{$\begin{array}{c}d \\
(\mathrm{~mm})\end{array}$} & \multirow{2}{*}{$\begin{array}{c}f_{c} \\
(\mathrm{MPa})\end{array}$} & \multirow{2}{*}{$\begin{array}{l}\rho \\
\% \\
\end{array}$} \\
\hline & $c_{\min }$ & $c_{m a ́ x}$ & & & & \\
\hline L1 & 250,00 & 250,00 & 1,00 & 94,00 & 29,00 & 1,39 \\
\hline L2 & 230,00 & 270,00 & 1,17 & 93,00 & 29,00 & 1,40 \\
\hline L3 & 215,00 & 285,00 & 1,33 & 94,00 & 29,00 & 1,39 \\
\hline L4 & 200,00 & 300,00 & 1,50 & 90,00 & 29,00 & 1,45 \\
\hline L5 & 165,00 & 335,00 & 2,03 & 91,00 & 22,00 & 1,43 \\
\hline L6 & 125,00 & 375,00 & 3,00 & 91,00 & 22,00 & 1,43 \\
\hline L7 & 110,00 & 390,00 & 3,55 & 91,00 & 22,00 & 1,43 \\
\hline L8 & 100,00 & 400,00 & 4,00 & 94,00 & 22,00 & 1,39 \\
\hline
\end{tabular}

Os perímetros dos pilares são constantes e iguais a $1000 \mathrm{~mm}$;

A altura efetiva $d$ foi obtida através da observação de pedaços de concreto extraídos dos centros das lajes. Estas medidas têm uma precisão de $\pm 2 \mathrm{~mm}$. A altura efetiva média é a distância entre a borda inferior da laje e o centro de gravidade das armaduras de flexão principais.
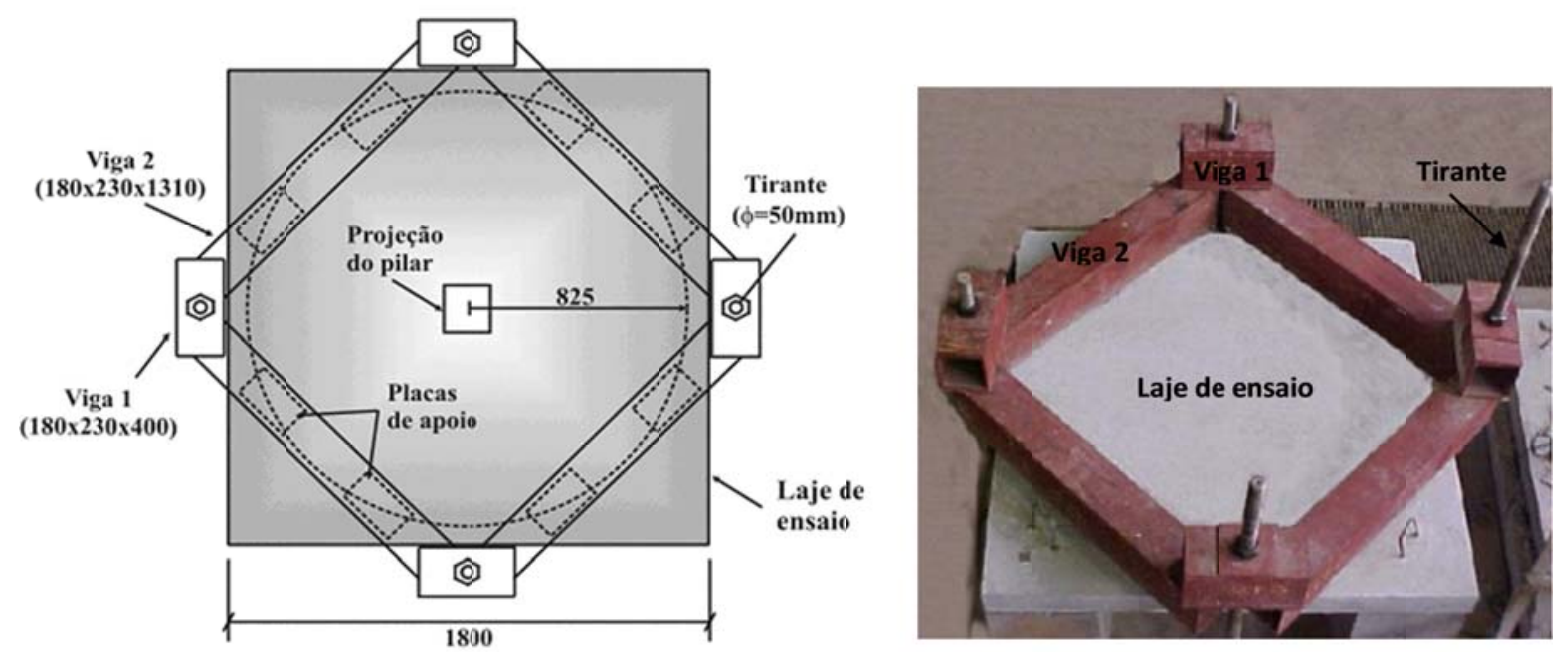

Figura 4: Sistema de ensaio e fotografia da laje - vista superior - (un idade em $\mathrm{mm}$ ).
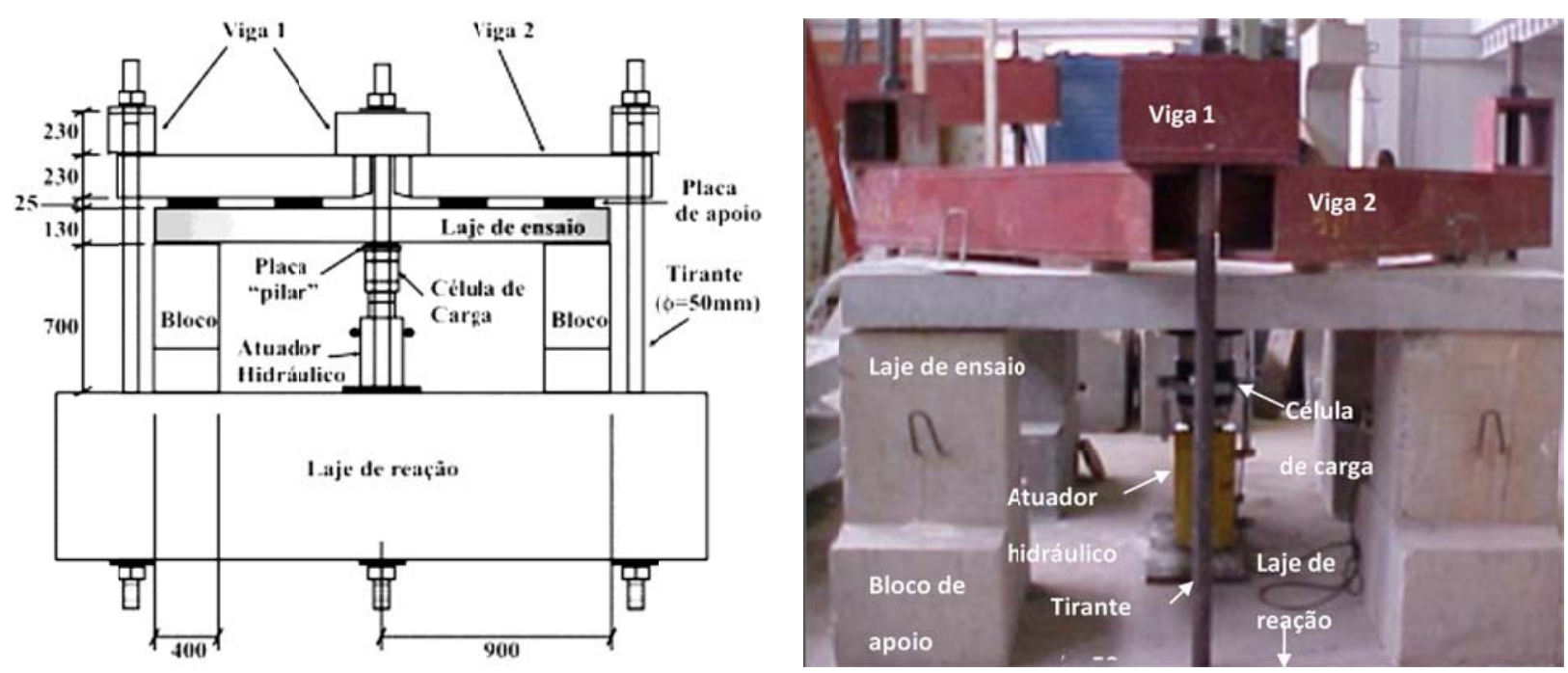

Figura 5: Sistema de ensaio e fotografia da laje - vista lateral - (unidade em $\mathrm{mm}$ ). 


\section{APRESENTAÇÃO E ANÁLISE DOS RESULTADOS}

As idades e propriedades mecânicas, com valores médios dos resultados dos corpos-de-prova (CP's) do concreto são apresentadas na Tabela 2. $\mathrm{Na}$ Tabela 3 é apresentado um resumo das propriedades mecânicas dos aços utilizados na confecção da armadura de flexão, através de ensaios de tração axial realizados em amostras, de acordo com a NBR-6152 (1992).

Os deslocamentos verticais aumentaram com o acréscimo do carregamento, sendo que os maiores valores obtidos foram medidos no ponto central da laje. A laje L1, que possui carregamento quadrangular, apresentou um perfil simétrico para os deslocamentos verticais nas direções $x$ e $y$. Nas lajes de carregamento retangular, os deslocamentos não se mostravam mais simétricos, com o aumento da relação $c_{\text {máx }} / c_{\text {min }}$, sendo $c_{\text {máx }}$ a maior dimensão, e $c_{\text {min }}$ a menor dimensão da seção transversal do pilar.

Nas lajes L6, L7 e L8 (lajes com índices de retangularidade iguais a $3,00,3,55$ e 4,00 ), os deslocamentos verticais na direção y, direção paralela à maior dimensão do pilar, foram superiores aos deslocamentos na direção $x$, todos equidistantes do centro da laje, devido à restrição imposta pelo pilar.

Os maiores pontos de deslocamento foram atingidos pelos pontos mais próximos ao centro da laje, sendo que, em todas as lajes, a armadura de flexão atingiu o escoamento em pontos próximos à face do pilar.

Foram realizadas medidas de deformações nas barras de aço da armadura de flexão, da borda superior, nas direções $x$ e $y$. Os maiores pontos de deformação foram atingidos pelos pontos mais próximos ao centro da laje, sendo que, em todas as lajes, a armadura de flexão atingiu o escoamento em pontos próximos à face do pilar.

As deformações das barras da armadura de flexão, medidas em relação à maior dimensão do pilar (direção y) foram superiores as obtidas em relação a menor dimensão (direção x), com exceção das lajes L1, L5 e L7, que apresentaram grandes deformações da armadura medidas em relação à menor dimensão do pilar, até atingir o escoamento.

As leituras realizadas nos extensômetros localizados na superfície inferior das lajes mostraram que não houveram deformações que comprometessem nesta região a integridade do concreto.

\section{Tabela 2: Propriedades mecânicas do concreto}

\begin{tabular}{|c|c|c|c|c|c|c|}
\hline Laje & $\begin{array}{l}\text { Idade } \\
\text { (dias) }\end{array}$ & $\begin{array}{c}f_{c} \\
(\mathrm{MPa})\end{array}$ & $\begin{array}{c}f_{c t, s p} \\
(\mathrm{MPa})\end{array}$ & $\begin{array}{c}E_{c} \\
(\mathrm{GPa})\end{array}$ & $\begin{array}{c}\mathrm{E}_{\mathrm{c}(\mathrm{NBR} / 03)} \\
(\mathrm{GPa})\end{array}$ & $\begin{array}{c}E_{c} / \\
E_{c(N B R / 03)}\end{array}$ \\
\hline \multirow{6}{*}{$\begin{array}{c}\mathrm{L} 1, \mathrm{~L} 2, \mathrm{~L} 3 \mathrm{e} \\
\mathrm{L} 4\end{array}$} & 7,00 & 21,10 & 2,30 & $*$ & 25,80 & - \\
\hline & 14,00 & 24,60 & 2,60 & $*$ & 27,80 & - \\
\hline & 21,00 & 27,50 & 2,70 & * & 29,40 & - \\
\hline & 28,00 & 29,00 & 3,40 & 28,70 & 30,10 & 0,95 \\
\hline & 30,00 & 29,60 & $*$ & $*$ & 30,50 & - \\
\hline & 41,00 & 29,60 & $*$ & $*$ & 30,50 & - \\
\hline \multirow{5}{*}{$\begin{array}{c}\mathrm{L} 5, \mathrm{~L} 6, \mathrm{~L} 7 \mathrm{e} \\
\mathrm{L8}\end{array}$} & 7,00 & 16,60 & $*$ & $*$ & 22,80 & - \\
\hline & 14,00 & 21,10 & $*$ & $*$ & 25,70 & - \\
\hline & 21,00 & 21,90 & * & * & 26,20 & - \\
\hline & 28,00 & 22,30 & 2,10 & 19,70 & 26,50 & 0,74 \\
\hline & 34,00 & 22,80 & * & * & 26,70 & - \\
\hline
\end{tabular}

* ensaio não realizado

Obs.: As lajes L1 e L2 foram ensaiadas com o concreto na idade de 28 dias, a laje L3 foi aos 30 dias e a L4 aos 41 dias. As lajes L5 a L8 foram ensaiasdas no período entre 28 e 34 dias. 
Tabela 3: Propriedades mecânicas dos aços

\begin{tabular}{cccccc}
$\begin{array}{c}\text { Diâmetro } \\
(\mathbf{m m})\end{array}$ & Lajes & $\begin{array}{c}\text { Área } \\
\left(\mathbf{m m}^{2}\right)\end{array}$ & $\begin{array}{c}f_{y k} \\
(\mathrm{MPa})\end{array}$ & $\begin{array}{c}E_{s} \\
(\mathrm{GPa})\end{array}$ & $\begin{array}{c}\boldsymbol{\varepsilon}_{y k} \\
(\mathrm{~mm} / \mathrm{m})\end{array}$ \\
\hline 6,30 & \multirow{2}{*}{ L1 a L8 } & 32,20 & 600,00 & 203,00 & 2,96 \\
12,50 & & 122,70 & 597,00 & 215,00 & 2,78 \\
\hline
\end{tabular}

As fissuras surgiram na superfície superior da laje e se desenvolveram de forma semelhante em todas as lajes. As fissuras radiais foram as primeiras a surgirem, em todas as lajes, ao redor do pilar e se propagaram em direção às bordas da laje com o aumento do carregamento. Estas fissuras apareceram com uma carga de $100 \mathrm{kN}$ nas lajes, estando entre $25 \%$ e $33 \%$ da carga de ruptura, exceto na laje $\mathrm{L} 8$, cujas fissuras surgiram sob uma carga de $75 \mathrm{kN}$, correspondendo a $27 \%$ da carga de ruptura.

As fissuras circunferenciais surgiram no carregamento de $100 \mathrm{kN}$, correspondendo ao intervalo entre $26 \%$ e $36 \%$ da carga de ruptura, exceto nas lajes L2, L3 e L4, que surgiram na carga de $125 \mathrm{kN}$, em torno de $32 \%$ da carga de ruptura, sendo que estas fissuras ligam-se nas fissuras radiais existentes e circundam 0 pilar. As Figuras 6 e 7 apresentam a fissuração das lajes L1 e L8, respectivamente.

A Tabela 4 e a Figura 8 apresentam um resumo das comparações entre as cargas de ruptura experimentais e as estimadas pelas normas de projeto. Os resultados mostram que a estimativa do $\mathrm{ACl} 318$ (2002) forneceu os resultados mais conservadores, com uma média de 1,82 para $V_{\text {exp }} / V_{\text {cal }}$ obtendo também o maior valor para o desvio padrão igual a 0,17. O Eurocode 2 (2001) apresentou o maior coeficiente de variação, sendo 9,86\%. O CEB-FIP MC90 (1991) e a NBR 6118 (ABNT, 2003) apresentaram praticamente o mesmo resultado para a média e os menores valores para o desvio padrão, e tiveram o coeficiente de variação igual a $9,75 \%$

A distribuição das forças cortantes em torno dos pilares pode influenciar na resistência última das lajes. Levando-se em conta o fenômeno da polarização das forças cortantes e a importância de utilizar a relação $c_{\text {máx }} / c_{\text {min }}$ nas análises das normas e códigos de projeto, os resultados experimentais da presente pesquisa e os resultados da literatura de lajes solicitadas nas duas direções foram utilizados para aproximar os resultados das expressões do ACI 318 (2002), CEB-FIP MC90 (1991), EUROCODE 2 (2001) e da NBR 6118 (ABNT, 2003) aos resultados reais.

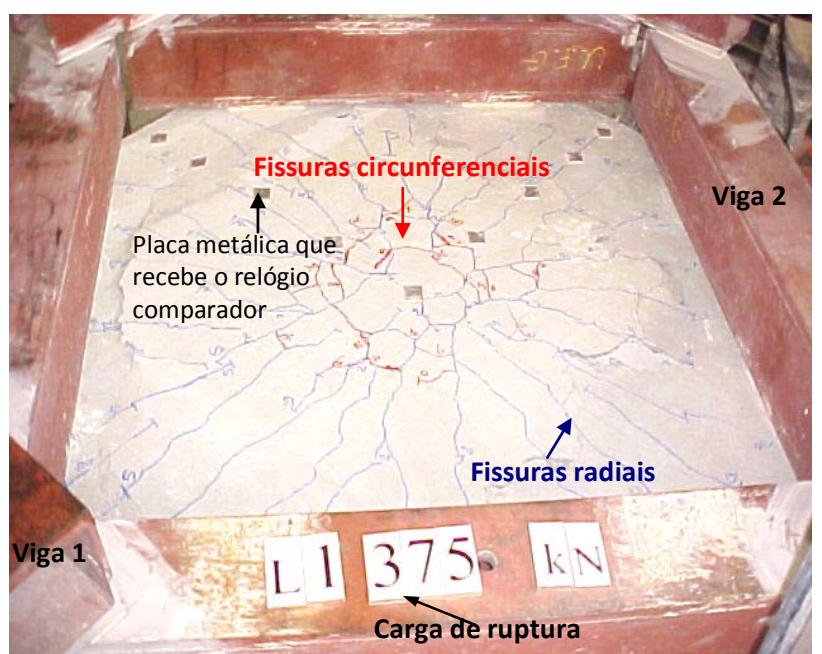

Figura 6: Fissuração da Laje L1

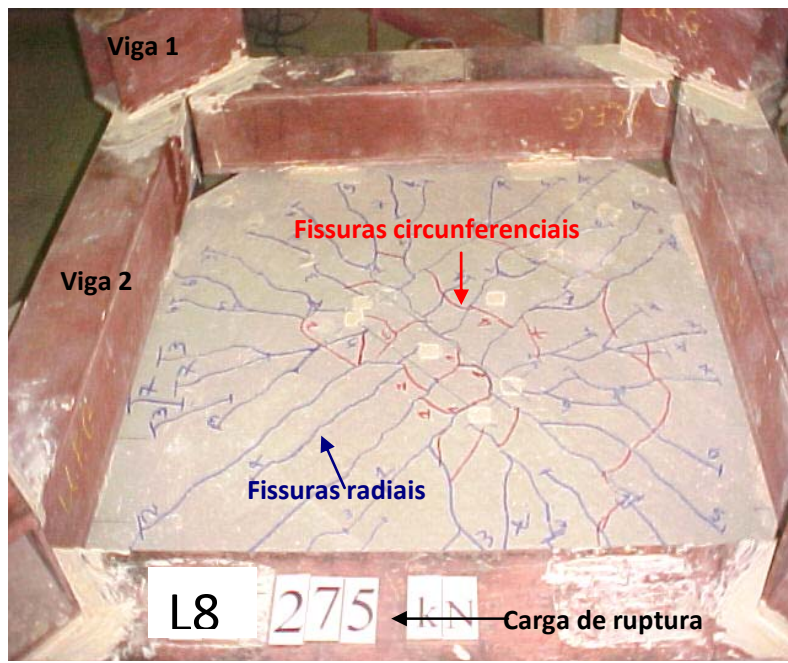

Figura 7: Fissuração da Laje L6

Para obter uma função real que passe mais próximo dos pontos $(x, y)$, que são os resultados experimentais das lajes da presente pesquisa e da literatura, sendo $\mathrm{x}$, os valores de $c_{\text {máx }} / c_{\text {minn }}$ e y, os valores para $V_{\text {exp }} / V_{\text {cal }}$ para um total de 53 lajes, utilizou-se o método dos mínimos quadrados, como apresenta a Tabela 5.

A Tabela 6 mostra os valores obtidos através das expressões do método dos mínimos quadrados, para índices de retangularidade entre 1 e 5.0 parâmetro " $\mu$ " deve ser incorporado diretamente na expressão para o 
cálculo da resistência ao puncionamento das normas como indicados na Tabela 7, $\mathrm{V}_{\text {cal,ef }}=\mu \mathrm{V}_{\text {cal }}$, sendo $\mathrm{c}_{\text {mín }} \mathrm{e}$ $c_{\text {máx }}$ a menor e maior dimensão do pilar.

O parâmetro " $\mu$ " para a norma do $\mathrm{ACl} 318$ (2002), CEB-FIP MC90 (1991) e NBR 6118 (ABNT, 2003) foram iguais a 1,47, 0,96 e 0,95, respectivamente, para índices de retangularidade entre 1 e 5, conforme apresentado na Tabela 7. Para índices entre 1 e 3, propõe-se para o EUROCODE 2 (2001) o parâmetro " $\mu$ " igual a 1,21, e para índices acima de 3 até 5 , considera “ $\mu$ ” igual a 1,02.

Tabela 4: Resumo da relação $V_{\text {exp }} / V_{\text {cal }}$ das lajes ensaiadas (normas e códigos)

\begin{tabular}{|c|c|c|c|c|c|c|c|}
\hline Laje & $\begin{array}{c}c_{\min } \\
(\mathrm{mm})\end{array}$ & $\begin{array}{c}c_{\text {máx }} \\
(\mathrm{mm})\end{array}$ & $\begin{array}{l}V_{\text {exp }} \\
\text { (kN) }\end{array}$ & $V_{\text {exp }} / V_{A C I}$ & $V_{\text {exp }} / V_{C E B}$ & $V_{\text {exp }} / V_{E C 2}$ & $V_{\text {exp }} / V_{N B R}$ \\
\hline L1 & 250,00 & 250,00 & 375,00 & 1,62 & 1,21 & 1,48 & 1,19 \\
\hline L2 & 230,00 & 270,00 & 390,00 & 1,70 & 1,27 & 1,56 & 1,25 \\
\hline L3 & 215,00 & 285,00 & 375,00 & 1,62 & 1,21 & 1,48 & 1,19 \\
\hline L4 & 200,00 & 300,00 & 395,00 & 1,80 & 1,32 & 1,65 & 1,31 \\
\hline L5 & 165,00 & 335,00 & 385,00 & 1,90 & 1,34 & 1,64 & 1,32 \\
\hline L6 & 125,00 & 375,00 & 350,00 & 2,15 & 1,26 & 1,57 & 1,25 \\
\hline L7 & 110,00 & 390,00 & 300,00 & 1,96 & 1,08 & 1,35 & 1,07 \\
\hline L8 & 100,00 & 400,00 & 275,00 & 1,80 & 0,96 & 1,19 & 0,95 \\
\hline \multicolumn{4}{|c|}{ Média } & 1,82 & 1,21 & 1,49 & 1,19 \\
\hline \multicolumn{4}{|c|}{ Desvio Padrão } & 0,17 & 0,12 & 0,15 & 0,12 \\
\hline \multicolumn{4}{|c|}{ Coeficiente de Variação (\%) } & 9,37 & 9,75 & 9,86 & 9,75 \\
\hline
\end{tabular}

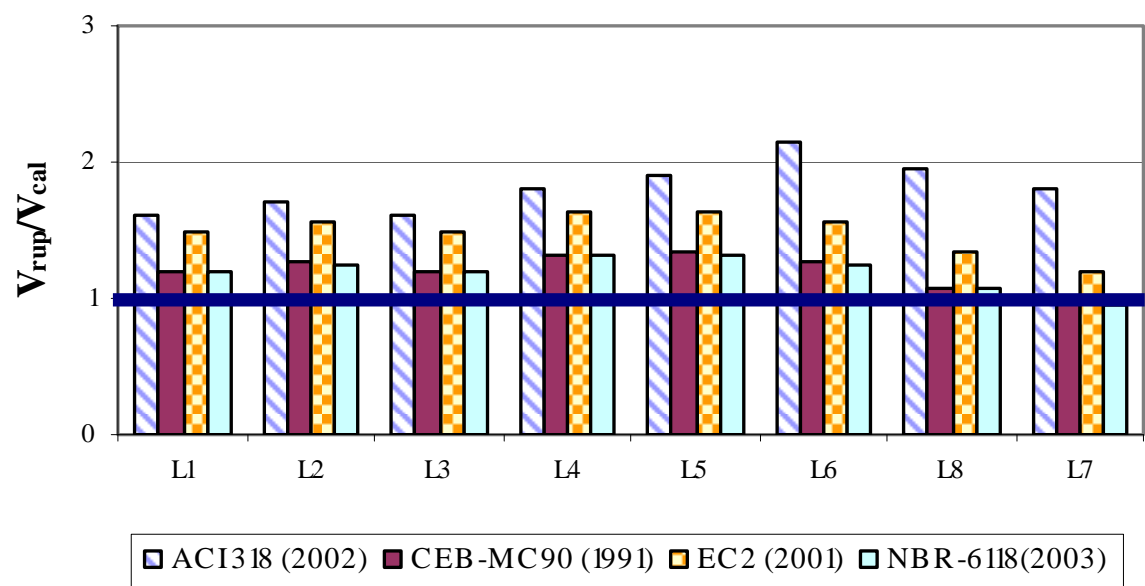

Figura 8: Gráfico da relação entre as cargas de ruptura e as cargas estimadas.

Tabela 5: Método dos mínimos quadrados

\begin{tabular}{cccc} 
Norma & \multicolumn{2}{c}{$y$} \\
\hline ACI 318 (2002) & $-0,0076 c_{\text {máx }} / c_{\text {min }}+1,4935$ \\
CEB-FIP MC90 (1991) & $-0,0553 \quad c_{\text {máx }} / c_{\text {min }}+1,1632$ \\
EURODODE 2 (2001) & $-0,0747 \quad c_{\text {máx }} / c_{\text {min }}+1,3957$ \\
NBR 6118 (ABNT, 2003) & $-0,0554 \quad c_{\text {máx }} / c_{\text {min }}+1,1538$ \\
\hline
\end{tabular}


Tabela 6: Valores obtidos das expressões do método dos mínimos quadrados

\begin{tabular}{ccccc}
$c_{\text {máx }} / c_{\text {mín }}$ & Prop_ACl/02 & Prop_CEB/91 & Prop_EC2/01 & Prop_NBR/03 \\
\hline 1,00 & 1,49 & 1,11 & 1,32 & 1,10 \\
2,00 & 1,48 & 1,05 & 1,25 & 1,04 \\
3,00 & 1,47 & 1,00 & 1,17 & 0,99 \\
4,00 & 1,46 & 0,94 & 1,10 & 0,93 \\
5,00 & 1,46 & 0,89 & 1,02 & 0,88 \\
\hline
\end{tabular}

\section{Tabela 7: Valores do parâmetro " $\mu$ " para as normas de projeto}

\begin{tabular}{|c|c|c|c|c|}
\hline$c_{\text {máx }} / c_{\text {min }}$ & Prop_ACI/02 & Prop_cEB/91 & Prop_ec2/01 & Prop_NBR/03 \\
\hline 1,00 & \multirow{5}{*}{1,47} & \multirow{3}{*}{1,05} & \multirow{4}{*}{1,21} & \multirow{3}{*}{1,05} \\
\hline 2,00 & & & & \\
\hline 3,00 & & & & \\
\hline 4,00 & & \multirow{2}{*}{0,90} & & \multirow{2}{*}{0,90} \\
\hline 5,00 & & & 1,02 & \\
\hline
\end{tabular}

Foi feito um comparativo do parâmetro " $\mu$ "e, percebe-se que a inclusão do parâmetro " $\mu$ " nas expressões de cálculo do ACI 318 (2002) e NBR 6118 (ABNT, 2003) resultaram em uma média para a relação $V_{\text {exp }} / V_{\text {cal }}$ igual a 1 , e as médias do EUROCODE 2 (2001) e CEB-FIP MC90 (1991) foram iguais a 1,01, com menores valores do desvio padrão para o CEB-FIP MC90 (1991) e NBR 6118 (ABNT, 2003), em relação às estimativas das expressões das normas citadas, estando a favor da segurança.

A inclusão do parâmetro " $\mu$ ” na equação do CEB-FIP MC90 (1991) conduziu a uma estimativa média para a relação $V_{\text {exp }} / V_{c a l}$ igual a 1,01. A inclusão do parâmetro " $\mu$ " na equação do $\mathrm{ACl} 318$ (2002) conduziu a uma estimativa média para a relação $\mathrm{V}_{\text {exp }} / \mathrm{V}_{\text {cal }}$ igual a 1 . A proposta estimou o menor desvio padrão, igual a 0,19, estando a favor da segurança.

\section{CONCLUSÕES}

As conclusões principais obtidas neste estudo foram as seguintes:

1. À medida que o índice de retangularidade aumentava, a resistência ao puncionamento e a resistência à flexão diminuíam. Todas as lajes romperam por punção.

2. A NBR 6118 (ABNT, 2003) forneceu resultados próximos ao do CEB-FIP MC90 (1991), sendo que os melhores índices de resistência foram obtidos pela norma brasileira. O ACl 318 (2002) mostrou-se mais conservador, com maiores valores da média e do desvio padrão.

3. A proposta do parâmetro " $\mu$ " nas normas analisadas apresentou melhores resultados para a resistência ao puncionamento, considerando a influência da relação $c_{\text {máx }} / c_{\text {min }}$ nas expressões dessas normas.

\section{AGRADECIMENTOS}

À Capes, ao Procad - Programa Nacional de Cooperação Acadêmica pelo apoio financeiro, e CNPq pelo suporte financeiro. Às empresas Realmix Concreto, Impercia - Produtos Químicos e Impermeabilizantes para Construções; Perfinasa - Perfilados e Ferros Nossa Senhora Aparecida Ltda; e ao Laboratório Carlos Campos Consultoria. 


\section{REFERÊNCIAS BIBLIOGRÁFICAS}

ACI Committee 318 (2002). Building Code Requirements for Reinforced Concrete ( $\mathrm{ACl} 318-02$ ) and Commentary ( $\mathrm{ACl}$ 318R-02). American Concrete Institute, Farmington Hills, Michigan, 2002, $391 \mathrm{pp}$.

AL-YOUSIF, A.T., REGAN, P.E. Punching resistances of RC slabs supported by large an/or elongated columns. The Structural Engineer, March, 2003, p. 130-134.

ASSOCIAÇÃO BRASILEIRA DE NORMAS TÉCNICAS. NBR 6118: Projeto de Obras de Concreto Armado - Procedimento. Rio de Janeiro, 2003.

NBR-6152/92: Materiais metálicos Determinação das propriedades mecânicas à tração Método de ensaio. Rio de Janeiro, 1992.

BORGES, L.L.J. (2004). Comportamento ao Puncionamento de Lajes Cogumelo de Concreto Armado com Pilares Retangulares e Furos de Grandes Dimensões. Tese de Doutorado, Publicação E.TD-002A/04, Departamento de Engenharia Civil e Ambiental, Universidade de Brasília, Brasília, DF, $367 \mathrm{p}$.

CEB-FIP MC90 (1991). CEB-FIP Model Code 1990: Final Draft . Bulletin D'Information 204, Committe EuroInternational du Beton, Lousanne, July 1991.

CORDOVIL, F. A. B. Punção em Placas de Concreto Armado. Tese de Doutorado. Departamento de Engenharia de Estruturas e Fundações. Escola Politécnica da Universidade de São Paulo, São Paulo, 1995, 393 p.

EUROCODE 2 (1992). Design of concrete structures - Part1: General Rules and Rules for Buildings. European Prestandard ENV 1992-1-1:1991. Comité Europeén de Normalisation, Brussels, 1992, 253 pp.

HAWKINS, N.M., FALLSEN, H.B., HINOJOSA, R.C. (1971). Influence of Column Rectangularity on the Behavior of Flat Plate Structures. ACl Publication SP-30: Cracking, Deflection, and Ultimate Load of Concrete Slab Systems, American Concrete Institute, Detroit, Michigan, 1971, p. 126-146.

OLIVEIRA, D.R.C. (2003). Análise experimental de lajes cogumelo de concreto de alta resistência com pilares retangulares. Tese de Doutorado em Estruturas e Construção Civil. Universidade de Brasília, Brasília, Agosto 2003, 183 pp.

REGAN, P.E., REZAI-JORABI,H. Shear resistance of one-way slabs under concentrated loads. ACl Structural Journal, March-April 1988, p.150-158.

SILVA, J.A. Punção em Lajes Cogumelo: Pilares Retangulares, Furos e Armadura de Cisalhamento. Dissertação de Mestrado em Engenharia Civil. Universidade Federal de Goiás, Goiânia, Julho 2003, 171 pp.

TENG, S., KUANG, K. L., CHEONG, H. K. (1999). Concrete Flat Plate Design - Findings of Joint BCA-NTU, R\&D Project, 1999, 15 pp. 